\title{
Comment on: 'Long-term impact of liver function on curative therapy for hepatocellular carcinoma: application of the ALBI grade'
}

\author{
Alessandro Cucchetti $^{*}, 1$, Matteo Cescon ${ }^{1}$ and Antonio Daniele Pinna ${ }^{1}$ \\ ${ }^{1}$ Department of Medical and Surgical Sciences, S.Orsola-Malpighi Hospital, Alma Mater Studiorum_University of Bologna, Bologna, Italy
}

Sir,

We read carefully the manuscript by Toyoda et al (2016) recently published in the British Journal of Cancer. They tried to verify whether ALBI score can provide sub-stratification, in terms of prognosis after resection, transplantation or ablation, of liver function in patients with early hepatocellular carcinoma. Results are surely of interest, but we believe that some issues need to be further discussed. One of the conclusions, emphasised in the abstract, was that ALBI grade-2 patients may, where the option exists, be more suitable for liver transplantation or the less invasive curative ablative therapies. However, as can be noted in Table 2 of the study by Toyoda et al. (2016), a survival advantage can be obtained after resection with respect to ablation also in the ALBI grade-2 class. In particular, by pooling the available data (random-effects model), a 5-year survival of $48.6 \%$ (95\% CI: 38.6-58.8) was calculated after resection and of $36.0 \%$ (95\% CI: 18.2-56.1) was obtained after ablation. Thus, even if inferior to figures of ALBI grade-1, resection still confers a longer survival in comparison with ablation. In addition, no data on tumour features (even if data are derived from early stages, a nonnegligible heterogeneity can be expected) for patients undergoing resection or ablation were provided, but only general surgical inclusion criteria were mentioned, making the comparison difficult to interpret with certainty. Thus, we believe that the Authors should be more cautious when suggesting ablation as an alternative option to resection for ALBI grade-2 patients as results, in terms of survival, do not support such proposal.

Despite this finding, we are in strong agreement of the suggestion for transplantation for ALBI grade-2, considering that the 5-year survival expected after liver transplant for early HCC largely exceeds $60 \%$, with even the possibility to provide the 'cure' from the tumour and the underlying liver disease (Cucchetti et al, 2014). The ALBI is surely of interest and when making recommendation for liver transplantation it would be of interest to compare its prognostic accuracy with that of the Model for End-stage Liver Disease (MELD) score, considering that the latter one is widely used as selection, and prioritization, criteria for liver transplantation (Toso et al, 2014). Can the ALBI score replace the MELD system?

In conclusion, we believe that results of the study by Toyoda et al (2016) can support the hypothesis that ALBI score can stratify prognosis among patients undergoing hepatectomy or ablation, but, as they are presented, cannot support to switch the option from surgical to nonsurgical therapies. An additional comparison with MELD performance would be of great interest.

\section{AUTHOR CONTRIBUTIONS}

AC, MC and ADP drafted the reply letter, provided their critical expertise and important intellectual contents, and shared the final version.

\section{CONFLICT OF INTEREST}

The authors declare no conflict of interest.

\section{REFERENCES}

Cucchetti A, Vitale A, Cescon M, Gambato M, Maroni L, Ravaioli M, Ercolani G, Burra P, Cillo U, Pinna AD (2014) Can liver transplantation provide the statistical cure? Liver Transpl 20: 210-217.

Toso C, Mazzaferro V, Bruix J, Freeman R, Mentha G, Majno P (2014) Toward a better liver graft allocation that accounts for candidates with and without hepatocellular carcinoma. Am J Transplant 14: 2221-2227.

Toyoda H, Lai PB, O’Beirne J, Chong CC, Berhane S, Reeves H, Manas D, Fox RP, Yeo W, Mo F, Chan AW, Tada T, Iñarrairaegui M, Vogel A, Schweitzer N, Chan SL, Sangro B, Kumada T, Johnson PJ (2016) Long-term impact of liver function on curative therapy for hepatocellular carcinoma: application of the ALBI grade. Br J Cancer 114: 744-750.

This work is published under the BJC's standard license to publish agreement. After 12 months the license terms will change to a Creative Commons AttributionNonCommercial-Share Alike 4.0 Unported License. 